University of Nebraska - Lincoln

DigitalCommons@University of Nebraska - Lincoln

P. F. (Paul Frazer) Williams Publications

Electrical \& Computer Engineering, Department

August 1974

\title{
Optical excitation of plasmons in a quasi-one-dimensional conductor
}

P. F. Williams

University of Nebraska - Lincoln, pfw@moi.unl.edu

M. A. Butler

Bell Laboratories, Murray Hill, New Jersey

D. L. Rousseau

Bell Laboratories, Murray Hill, New Jersey

Follow this and additional works at: https://digitalcommons.unl.edu/elecengwilliams

Part of the Electrical and Computer Engineering Commons

Williams, P. F.; Butler, M. A.; and Rousseau, D. L., "Optical excitation of plasmons in a quasi-onedimensional conductor" (1974). P. F. (Paul Frazer) Williams Publications. 28.

https://digitalcommons.unl.edu/elecengwilliams/28

This Article is brought to you for free and open access by the Electrical \& Computer Engineering, Department of at DigitalCommons@University of Nebraska - Lincoln. It has been accepted for inclusion in P. F. (Paul Frazer) Williams Publications by an authorized administrator of DigitalCommons@University of Nebraska - Lincoln. 


\title{
Optical excitation of plasmons in a quasi-one-dimensional conductor
}

\author{
P. F. Williams, M. A. Butler, and D. L. Rousseau \\ Bell Laboratories, Murray Hill, New Jersey 07974 \\ Aaron N. Bloch* \\ Department of Chemistry, The Johns Hopkins University, Baltimore, Maryland 21218 \\ (Received 4 March 1974)
}

\begin{abstract}
We have carefully determined the optical spectrum of $\mathrm{K}_{2} \mathrm{Pt}(\mathrm{CN})_{4} \mathrm{Br}_{0.3} \cdot 3 \mathrm{H}_{2} \mathrm{O}$ near the reflectivity edge. Our data reveal, in addition to the expected monotonic background, a broad peak in $\epsilon_{2}(\omega)$ above a threshold coinciding with the zero of $\epsilon_{1}(\omega)$ near $16000 \mathrm{~cm}^{-1}$. We assign this band to indirect excitation of plasmons. Details of the excitation of plasmons are discussed with particular attention being paid to the problems presented by the one-dimensional character of the material. It is concluded that the electronic structure of this material cannot be adequately described by either a free-electron model or a one-band tight-binding model.
\end{abstract}

The "mixed-valence" cyanoplatinates are members of a class of materials exhibiting high electrical conductivity in one dimension. ${ }^{1}$ The static and low-frequency electronic properties of these materials are not those of simple metals. Instead, they are subject to the severe constraints imposed upon the electron gas by instabilities ${ }^{2}$ and disorder. ${ }^{3}$ It is clear, however, that at sufficiently high frequencies the conduction electrons must behave as though they are metallic in one dimension. For example, dispite the rather complicated infrared spectrum of $\mathrm{K}_{2} \mathrm{Pt}(\mathrm{CN})_{4} \mathrm{Br}_{0.3} \cdot 3 \mathrm{H}_{2} \mathrm{O}$, in the visible the reflectivity for light polarized parallel to the chain axis exhibits a well-defined plasma edge ${ }^{4}$ at about $16000 \mathrm{~cm}^{-1}$.

We have undertaken a study of the optical properties in this region of the spectrum, with results that offer some insights into the dynamics of electrons in a quasi-one-dimensional metal. In particular, we observe a maximum ${ }^{5}$ in $\epsilon_{2}(\omega)$ qualitatively similar to the disorder-induced plasma absorption treated by Hopfield ${ }^{6}$ for the case of an isotropic metal. (This absorption process is not to be confused with the purely geometric coupling effects predicted by Bulaevskii and Kucharenko ${ }^{7}$ and observed by Brüesch. ${ }^{8}$ ) Explanation of this absorption in terms of excitation of plasmons by the Hopfield mechanism is considered with emphasis on the problems of extending the three-dimensional system to the one-dimensional case. An explanation of this absorption given by Wagner et al. ${ }^{9}$ is discussed.

We have measured both the reflection spectrum and the optical absorption for $\vec{E}$ parallel to the chain axis in order to determine precisely the parallel components of $\epsilon_{1}(\omega)$ and $\epsilon_{2}(\omega)$ in the range $10000-24500 \mathrm{~cm}^{-1}$. For the reflectivity studies, we grew crystals $1-\mathrm{mm}$ thick and up to $1-\mathrm{cm}$ long by slow evaporation from aqueous solution. Because of the very high-absorption coefficient for light polarized parallel to the needle axis, however, we were unable to polish a crystal thin enough for transmission experiments. Therefore the absorption measurements were performed on samples prepared by painting a glass slide with a saturated solution of the compound. This procedure produced needles about $10-\mu \mathrm{m}$ wide and $0.5-1-\mu \mathrm{m}$ thick. To study these small samples, a microscope optical system was used to project an image of the crystal on the slit of the spectrometer.

Even with our thinnest samples a satisfactory absorption measurement could not be made below the reflectivity edge, and it was necessary to use a Kramers-Kronig analysis ${ }^{10}$ to extend $\epsilon_{1}^{\prime \prime}$ and $\epsilon_{2}^{\prime \prime}$ into this region. This analysis requires knowledge of the absorption at some point. For this purpose we chose the frequency $23000 \mathrm{~cm}^{-1}$, where we found an absorption coefficient of $2500 \mathrm{~mm}^{-1}$, more than an order of magnitude larger than reported by Yamada. ${ }^{11}$

The general features of our results are consistent with those of Ref. 4 and 9. The real part of the dielectric constant passes through zero near $16000 \mathrm{~cm}^{-1}$ and the imaginary part shows a behavior similar to the $\omega^{-3}$ Drude behavior. Further, our data show clearly an additional broad pronounced peak in $\epsilon_{2}(\omega)$ centered around $18000 \mathrm{~cm}^{-1}$. Figure 1 shows a plot of $\epsilon_{2}(\omega)$. The dashed line in the figure is an $\omega^{-3}$ extrapolation of the monotonic background, included for reference. Our data are insufficient to distinguish between an $\omega^{-3}$ Drude dependence for this background and other predicted functional dependences. ${ }^{9,12}$ While the possibility that the peak results from an interband transition cannot be entirely dismissed, the proximity of the absorption threshold to the zero of $\epsilon_{1}(\omega)$ at $16000 \mathrm{~cm}^{-1}$ certainly suggests the alternate possibility that the light field has been coupled to plasmon excitations. ${ }^{13}$ 


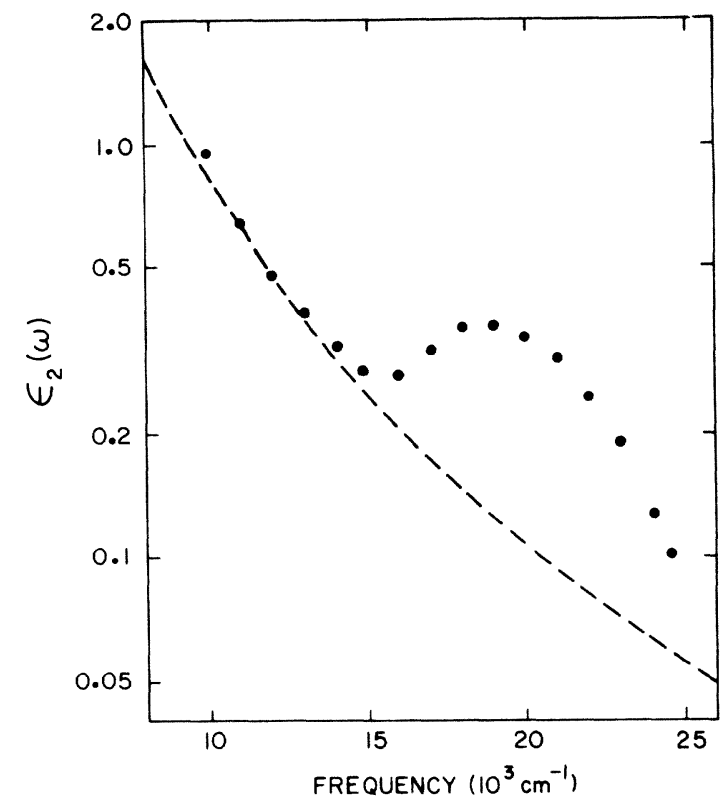

FIG. 1. Imaginary part of the dielectric constant parallel to the chain direction determined from a KramersKronig analysis (points). The dashed curve is an $\omega^{-3}$ extrapolation of the monotonic background included for reference.

In a three-dimensional electron gas, the transverse electromagnetic radiation field cannot couple directly to the longitudinal plasma oscillations. The material under consideration, however, is more accurately represented as a periodic array of parallel one-dimensional metallic filaments. In such a system, the constraint that electrons be localized on the strands imparts some transverse character to charge oscillations propagating at oblique angles to the chain axis, and direct coupling to the light field is possible in certain optical geometries. ${ }^{7,8}$ Such a coupling is precluded, however, when the light is at precisely normal incidence and polarized along the chain axis, ${ }^{7,8}$ as in our experiments.

We conclude that to explain our data requires an additional coupling mechanism. Hopfield ${ }^{6}$ has studied the optical response of an isotropic threedimensional metal in detail. He finds that when dynamical-screening effects are included, scattering by phonons or static disorder induces, in addition to the Drude absorption, an indirect excitation of plasmons. His arguments may be easily modified to apply to a model system composed of a set of linear chains to which the electrons are tightly localized. If the optical electric field is along the chain axis, the imaginary part of the dielectric constant to second order in the Fourier component $V_{\vec{Q}}$ of the scattering potential is given by

$$
\epsilon_{2}(\omega)=\frac{1}{m^{2} \omega^{4}} \sum_{\vec{Q}}\left|V_{\vec{Q}}\right|^{2} Q^{2} q^{2} \operatorname{Im} \frac{1}{\epsilon_{\vec{Q}}(\omega)},
$$

where $q$ is the component of the wave vector $\vec{Q}$ along the chain axis,

$$
\epsilon_{\vec{Q}}(\omega)=\left(1 / Q^{2}\right) \vec{Q} \cdot \epsilon(\vec{Q}, \omega) \cdot \vec{Q},
$$

and $\epsilon(\vec{Q}, \omega)$ is the dielectric tensor in the absence of impurity scattering. The plasmon contributions to $\epsilon_{2}(\omega)$ are manifested through the poles of $\operatorname{Im}[1 /$ $\left.\epsilon_{\vec{Q}}(\omega)\right]$.

Strictly speaking, Eq. (1) is valid only for wavelengths much larger than a lattice spacing since at long wavelengths local-field effects need not be considered. The inclusion of local-field effects simply introduces the possibility of Umklapp processes so that the components $V_{\vec{Q}+\vec{G}}$, where $\vec{G}$ is a reciprocal-lattice vector, may contribute to the creation of plasmons of wave vector $\vec{Q}$. The form of (1) will remain essentially unchanged. Although there are some difficulties, it appears that the Hopfield mechanism is capable of adequately explaining the observed peak in $\epsilon_{2}(\omega)$.

Wagner et al. ${ }^{9}$ have proposed an alternative mechanism which results from the creation of two excitations, one a plasmon and the other an intraband electron-hole pair. It has been pointed out, ${ }^{6,14}$ however, that because momentum is conserved in a translationally invariant system, such a process cannot contribute to a real absorption of energy. Since over-all momentum is conserved, the current is not damped, and there can be no loss of energy. A momentum sink is required in order to change the total current. Intraband electron-electron interactions will not suffice.

The crystalline lattice may be employed as the momentum sink. In an extended-zone scheme, an electron-scattering process in which the electron wave vector is changed by a reciprocal-lattice vector would correspond to an interband transition. Thus the mechanism of Wagner et al. would be allowed if the electron-hole excitation produced consists of an electron in one band and the hole in another. As their mechanism stands, however, without a lattice interaction, it is incorrect. Crystalline irregularities resulting from either phonons or lattice defects may also serve as momentum sinks. For this mechanism, ${ }^{6}$ the simultaneous production of an electron-hole pair is not required.

Regardless of the details of the process involved, any attempt to interpret the peak in $\epsilon_{2}(\omega)$ in terms of plasma excitations is troubled by the propagation-angle dependence of the plasmon frequency ${ }^{12}$ For long wavelengths, the plasmon frequency is proportional to $\cos \theta, \theta$ being the angle between the plasmon wave vector and the chain axis. ${ }^{12,15} \mathrm{Be}-$ cause there are more off-axis plasmon modes, the 
$\cos \theta$ dependence of the plasmon frequency does not result in a peak near the $\theta=0^{\circ}$ plasma frequency for these long-wavelength modes. The presence of the peak may be explained in terms of plasmons by either assuming a special form for $V_{\vec{Q}}$ which does not allow these off axis modes, or by considering the effects of shorter-wavelength plasmons, for which the angular frequency dependence is lost.

It is apparent that if we consider only the longerwavelength plasmon modes (for which the $\cos \theta$ dependence is maintained), $V_{\vec{Q}}$ must be zero unless $\vec{Q}$ is parallel to the chain axis for a well-defined peak in $\epsilon_{2}(\omega)$ to be observed. Unfortunately, we can at present only speculate on the nature of this scattering potential, but there is, in general, no selection rule restricting $V_{\vec{Q}}$ to be nonzero only for $\vec{Q}$ parallel to the chain axis. Such a special form for $V_{\vec{Q}}$ would require the scattering potential to be uniform in directions perpendicular to the chain axis. This form is obtained only for special cases. Electron scattering dominated by scattering from phonons propagating along the chain axis provides an example of such a special case. If the $\mathrm{Br}^{-}$or $\mathrm{K}^{+}$ ions occupy sites in a fashion such that there is a high degree of order in directions perpendicular to the chain axis, ${ }^{16}$ scattering from these ions could constitute another such special case. Unless $V_{\vec{Q}}$ has this special form, long-wavelength plasmons will not produce a well-defined onset of the peak in $\epsilon_{2}(\omega)$ at the $\theta=0^{\circ}$ plasma frequency.

In the absence of a special form for $V_{\vec{Q}}$ the peak may be explained by noting that the angle dependence of the plasmon frequency is lost as the plasmon wavelength becomes shorter. ${ }^{12}$ This observation combined with density -of -states considerations and the $Q^{2} q^{2}$ factor in Eq. (1) makes it possible to explain the absorption peak in terms of large $-q$ plasma excitations. Additionally, the giant Kohn anomoly ${ }^{2}$ may play an important role by providing a strongly increased electron-phonon interaction at $q=2 k_{F}$. Williams and Bloch ${ }^{12}$ have shown that within the random-phase approximation (RPA) (i) the plasmons are not Landau damped by the single-particle excitations, so that there is no cutoff wave vector such as Wagner et al. have suggested, and (ii) for a relatively narrow-band tight-binding one-dimensional electron gas, such as one might find in $\mathrm{K}_{2} \mathrm{Pt}(\mathrm{CN})_{4} \mathrm{Br}_{0.3} \cdot 3 \mathrm{H}_{2} \mathrm{O}$, the plasmon-dispersion curve is rather flat throughout the Brillouin zone. Such flatness is just what is needed to explain the observed absorption peak. Since a one-dimensional free-electron model results in a quadratic plasmon dispersion, ${ }^{12}$ such a model cannot produce a peak such as we observe.

There are a few caveats which must be kept in mind, however. First, the calculation of the plas mon dispersion by Williams and Bloch ${ }^{12}$ was carried out within the random-phase approximation.
It is by no means clear that this takes adequate account of the short-range electron-electron interaction. These effects are probably not important at long wavelengths, but for large $Q$ they can appreciably influence the plasmon dispersion.

Another interesting point involves the magnitude of the long-wavelength plasma frequency. Since it is a long wavelength excitation, the effects of electron correlation should not appreciably affect the calculated value for the $q=0$ plasma frequency. For propagation along the chain axis, this frequency should be given by $\omega_{p}^{2}=8 \pi e^{2} v_{F} / \epsilon_{\infty} \hbar$ where $\Re_{\text {is }}$ the number of strands per unit area, $e$ the electronic charge, $v_{F}$ the Fermi velocity, and $\epsilon_{\infty}$ the background dielectric constant. The only parameter not well known is $v_{F}$. Assuming a $\frac{5}{6}$ filled band and adjusting the Fermi velocity to get the observed plasma frequency, we find a bandwidth of $8.5 \mathrm{eV}$. This value is unreasonably large in light of the known optical properties and the expected overlap of the platinum $d$ bands. Using reasonable values for the parameters involved, it is very difficult to raise the predicted plasma frequency above $1 \mathrm{eV}$, whereas the measured value is $2 \mathrm{eV}$. Thus it appears that the system is more complicated than a simple one-band model.

A number of sources for this additional complication are possible. The platinum atoms on adjacent sites are not quite equivalent so that there are probably two closely spaced bands, one of which is the conduction band. In addition, the electronic structure of the material may be very complicated in the region within $2 \mathrm{eV}$ of the conduction band, so that transitions to other bands may be important.

Both the above considerations may significantly change the predicted plasma frequency and the shape and position of the absorption peak. In the absence of detailed knowledge about the electronic structure, we are not prepared, however, to calculate the precise form of $\epsilon_{2}(\omega)$ at this time.

The measurements presented here, which agree with measurements by other groups, ${ }^{4,9}$ and our examination of the data provide an initial step in illuminating the electronic structure of the quasione-dimensional conductors. We have seen that plasmons are the probable origin of the peak in $\epsilon_{2}$ near the plasma frequency, although the details of the mechanism are clouded by the complex nature of the plasmon modes. The theoretical treatment of these modes, given in the previous paper, ${ }^{12}$ combined with the measured plasma frequency indicates that a single-band tight-binding description is not appropriate and the band structure is probably more complex. These results point toward an examination of the role played by the empty $p_{2}$ band, the two platinum atoms per unit cell, and the admixture of other bands into the 
$5 d_{z^{2}}$ band, in determining the electronic structure of these materials.

We wish to thank S. T. Chui and C. M. Varma for many stimulating discussions and J. J. Hopfield for pointing out the importance of off-axis modes. We also thank $\mathrm{H}$. Wagner for sending us a preprint of his paper. Finally, A. N. B. is grateful to Bell Laboratories, Murray Hill, N.J. for their hospitality during part of the time that this work was in progress.
${ }^{*}$ Work at Johns Hopkins University supported by grants from the Advanced Research Projects Agency of the Department of Defense and from the National Science Foundation.

${ }^{1}$ For reviews, see K. Krogmann, Angew. Chem. 8,35 (1969); I. F. Schegolev, Phys. Status Solidi A $1 \overline{2}, 9$ (1972); H. R. Zeller, Festkörperprobleme 13, 31 (1973).

${ }^{2}$ B. Renker, H. Rietschel, L. Pintschovius, W. Glaser, P. Brüesch, D. Kuse, and M. J. Rice, Phys. Rev. Lett. 30,1144 (1973).

${ }^{3}$ A. N. Bloch, R. B. Weisman, and C. M. Varma, Phys. Rev. Lett. 28, 753 (1972).

${ }^{4} \mathrm{~J}$. Bernasconi, P. Brüesch, D. Kuse, and H. R. Zeller, Brown-Boveri Research Report No. KLR-73-05 (1973) (unpublished).

${ }^{5}$ This peak also has been seen by others. See Refs. 4 and 9.

${ }^{6}$ J. J. Hopfield, Phys. Rev. 137, A419 (1965).

${ }^{7}$ L. N. Bulaevskii and Yu. A. Kukhavenko, Sov. Phys. Solid State 14, 2076 (1973).
${ }^{8} \mathrm{P}$. Brüesch, Solid State Commun. 13,13 (1973).

${ }^{9}$ H. Wagner, H. P. Geserich, R. V. Baltz, and K. Krogman, Solid State Commun. 13, 659 (1973).

${ }^{10} \mathrm{P}$. F. Williams, thesis (University of Southern California, 1973) (unpublished), R. K. Ahrenkiel, J. Opt. Soc. Am. 61, 1651 (1971).

${ }^{11}$ Shoichiro Yamada, Nippon Kagaku Zasshi 86, 753 (1965).

${ }^{12} \mathrm{P}$. F. Williams and A. N. Bloch, preceding paper, Phys. Rev. B 10, 1097 (1974).

${ }^{13}$ Such effects have been observed in liquid $\mathrm{Hg}$. See $\mathrm{E}$. G. Wilson and S. A. Rice, Phys. Rev. 145, 55 (1966).

${ }^{14}$ The cancellation of diagrams responsible for the effect has been explicitly shown by A. Ron and N. Tzoar [Phys. Rev. 131, 12 (1963)]. See especially p. 16. See also P. A. Fedders, and P. C. Martin, Phys. Rev. 143,245 (1966).

${ }^{15}$ I. E. Dzyaloshinskii and E. I. Kats, Zh. Eksp. Teor. Fiz. 55, 338 (1968) [Sov. Phys.-JETP 28, 178 (1969)].

${ }^{16} \mathrm{~J}$. C. Inkson (unpublished). 